\section{Storage Characteristics of New Sweet Cherry Cultivars}

\author{
F. Kappel ${ }^{1}$, P. Toivonen, D.-L. McKenzie, and S. Stan \\ Agriculture and Agri-Food Canada, Pacific Agri-Food Research Centre, \\ Summerland, B.C. Canada VOH 1 ZO
}

Additional index words. Prunus avium, modified-atmosphere packaging, stem shrivel, stem browning, fruit surface pitting, firmness, fruit respiration

\begin{abstract}
Several sweet cherry (Prunus avium L.) cultivars were stored in air or modifiedatmosphere packages (MAP) at $1^{\circ} \mathrm{C}$ for 2 or 4 weeks, respectively. The new cultivars included 'Santina', 'Sumpaca Celeste', 'Sumnue Cristalina', 'Sumste Samba', 'Sandra Rose', 'Sumleta Sonata', and 'Skeena', and the standards were 'Lapins', 'Sweetheart', and 'Bing'. Fruit were rated for defects (stem browning, stem shrivel and fruit surface pitting), and fruit quality at harvest and after storage. Weight loss during storage was influenced by year, storage treatment, and cultivar. Stem shrivel, stem browning, and fruit surface pitting varied among cultivars and years. Generally, fruit stored in MAP had higher fruit firmness than at harvest or when stored in air. The respiration rate of fruit was lower in later than in earlier maturing cultivars, but respiration rate at harvest was not related to any of the quality measurements taken after storage.
\end{abstract}

The sweet cherry breeding program at Agriculture and Agri-Food Canada at Summerland has introduced many cultivars that have been adopted by sweet cherry growers around the world. New cultivars recently introduced from the program include 'Santina', 'Sumpaca Celeste', 'Sumnue Cristalina', 'Sumste Samba', 'Sandra Rose', 'Sumleta Sonata', and 'Skeena' (Kappel et al., 1998, 2000a, 2000b). They cover a range of maturities from $8 \mathrm{~d}$ before 'Van' to $\approx 15 \mathrm{~d}$ after 'Van', and all have large fruit size and good firmness. However, the postharvest quality and behavior of these new cultivars have not been determined.

The flavor of sweet cherries is largely determined by sugar content and acidity. During storage acidity changes more rapidly than does soluble solids (i.e., sugar) control (Drake and Fellman, 1987). Loss in flavor during modified-atmosphere (MA) storage is primarily associated with reduced acidity (Meheriuk et al., 1995). Cultivars may differ in either acidity at harvest or rate of change in acidity during storage.

Fruit firmness is also a very important postharvest quality factor. Cultivars differ in susceptibility to postharvest softening (Brown and Bourne, 1988). Other quality traits that can be affected by storage are fruit surface pitting and stem quality (browning and shrivel); Looney et al. (1996) noted cultivar differences in both of these characteristics.

Cherries have no starch reserves, therefore maintenance of respiration relies prima-

\footnotetext{
Received for publication 12 Sept. 2000. Accepted for publication 21 Dec. 2000. Contribution no. 2096 of the Pacific Agri-Food Research Centre. The financial assistance of the Washington State Tree Fruit Research Commission, Oregon Sweet Cherry Commission, and the Matching Investment Initiative program of Agriculture and Agri-Food Canada is greatly appreciated.

${ }^{1}$ To whom requests for reprints should be addressed. E-mail: kappelf@em.agr.ca.
}

rily upon organic acids (Singh et al., 1970). Seske (1988) reported that respiration rates differed among sweet cherry cultivars and speculated that this could provide an indication of storage potential.

The objectives of this study were to determine: 1) the storage characteristics of the fruit of new sweet cherry cultivars introduced by the breeding program; and 2) if high acidity and respiration rate at harvest were indicators of potential storage quality.

\section{Materials and Methods}

The cultivars evaluated in 1997 and 1998 were Santina, Celeste, Cristalina, Samba, Sandra Rose, and Bing (Group 1); and those evaluated in 1997, 1998, and 1999 were Sonata, Skeena, Lapins, Sweetheart and Bing (Group 2). Group 1 cultivars tended to mature early to midseason, whereas those in Group 2 tended to be midseason to later in maturing. 'Bing', the industry standard, was included with both groups. Fruit were carefully harvested in the morning and sorted and culled in the field. Each cultivar was harvested when it was judged to be at commercial maturity, based upon subjective assessment of skin and flesh color, along with taste 4 weeks of modified-atmosphere storage. (level of sweetness compared to acidity). None of the fruits studied was treated with gibberellic acid. Fruits were randomly placed into clear plastic clamshell containers [1.5 dry pint $(=775 \mathrm{~mL})$; Berigard Hinged Basket, Packaging Corporation of America, Northbrook., Ill.]. For each cultivar, 15 samples of $500 \mathrm{~g}$ each were prepared. Five were analyzed the day following harvest, five after 2 weeks of air storage and five after 4 weeks of storage in modified-atmosphere packages (MAP). All containers of fruit were stored at $1{ }^{\circ} \mathrm{C}$ and $80 \%$ relative humidity $(\mathrm{RH})$. The MAP bags used were made of Cryovac PD941 film (Cryovac, Duncan, S.C.). Fruit in clamshell containers were cooled overnight at $1{ }^{\circ} \mathrm{C}$, then placed inside the MAP bag and sealed. The atmosphere in the MAP bags was developed passively. For air storage the clamshells full of fruit were placed inside the cold storage room. The total weight of container and fruit was measured prior to storage and upon removal from storage.

For quality analysis, 25 fruits were randomly selected from the 500-g sample and first rated for surface pitting, stem shrivel, and stem browning using a four-point scale ( 4 = unblemished fruit or stems; 3 = slight defect; 2 = moderate defect; 1 = severe defect). The firmness of these 25 fruit was measured using a FirmTech 1 pressure tester (BioWorks, Stillwater, Okla.). Fruits from the firmness measurement were combined, the stems were removed, and the juice was expressed by crushing the fruits in a plastic bag. Total soluble solids concentration (TSSC) of the juice was measured using an Abbe Mark II refractometer (AO Scientific Instruments, Buffalo, N.Y.). Titratable acidity (TA) of a $10-\mathrm{mL}$ sample of juice was measured using a $719 \mathrm{~S}$ Titrino autotitrator (Metrohm, Herisau, Switzerland) and is expressed as milliliters of $0.1 \mathrm{~N} \mathrm{NaOH}$ required to bring the $\mathrm{pH}$ to 8.1 .

At harvest, fruit samples ( 15 to $30 \mathrm{~g}$ ) were placed in 1-L plastic jars with tight-fitting lids and put into a $20{ }^{\circ} \mathrm{C}$ incubator (model 307, Low Temperature Incubator; Fisher Scientific Canada, Nepean, Ont.). The cultivars evaluated were: 'Burlat', 'Celeste', 'Santina', 'Cristalina', 'Sandra Rose', 'Samba', 'Bing', 'Skeena', 'Sonata', '13N-07-70', 'Lapins', '13N-07-39', 'Sweetheart', 'Symphony', and 'Staccato'. They covered a range of maturity from mid- to late-June to early August. Jars

Table 1. Percentage of weight loss (\%) of Group 2 sweet cherry fruit after 2 weeks of air storage or

\begin{tabular}{lcccccccc}
\hline \hline \multirow{2}{*}{ Storage } & & \multicolumn{5}{c}{ Cultivar } & Storage & Year \\
\cline { 3 - 8 } & Year & Bing & Lapins & Skeena & Sonata & Sweetheart & means & means \\
\hline Air & 1997 & $6.4 \mathrm{~b}^{\mathrm{z}}$ & $2.2 \mathrm{gh}$ & $1.8 \mathrm{~h}$ & $2.7 \mathrm{fg}$ & $2.1 \mathrm{gh}$ & $4.5 \mathrm{~A}$ & $1.6 \mathrm{X}$ \\
& 1998 & $5.3 \mathrm{c}$ & $4.2 \mathrm{~d}$ & $3.8 \mathrm{de}$ & $5.3 \mathrm{c}$ & $3.1 \mathrm{ef}$ & & $2.6 \mathrm{Y}$ \\
& 1999 & $10.4 \mathrm{a}$ & $4.3 \mathrm{~d}$ & $6.8 \mathrm{~b}$ & $6.6 \mathrm{~b}$ & $3.0 \mathrm{f}$ & & $3.4 \mathrm{Z}$ \\
Modified & & & & & & & \\
atmosphere & 1997 & $0.5 \mathrm{j}$ & $0.4 \mathrm{j}$ & $0.0 \mathrm{k}$ & $0.3 \mathrm{j}$ & $0.1 \mathrm{j}$ & $0.6 \mathrm{~B}$ \\
& 1998 & $1.5 \mathrm{hi}$ & $0.3 \mathrm{i}$ & $0.8 \mathrm{ij}$ & $0.7 \mathrm{j}$ & $0.7 \mathrm{j}$ & & \\
& 1999 & $0.8 \mathrm{ij}$ & $0.7 \mathrm{j}$ & $0.7 \mathrm{j}$ & $0.7 \mathrm{j}$ & $0.5 \mathrm{j}$ & & \\
Cultivar means & & $4.2 \mathrm{E}^{\mathrm{y}}$ & $2.0 \mathrm{H}$ & $2.3 \mathrm{G}$ & $2.7 \mathrm{~F}$ & $1.6 \mathrm{I}$ & &
\end{tabular}

${ }^{2}$ Mean separation for cultivar-storage-year combinations (lowercase letters) by LSD, $P \leq 0.05$.

${ }^{\mathrm{y}}$ Mean separation for cultivars, storage types, or years (uppercase letters) by LSD, $P \leq 0.05$. 
Table 2. Stem and fruit defect ratings $\mathrm{s}^{\mathrm{z}}$ of sweet cherry cultivars in 1997 and 1998 at harvest, after 2 weeks of air storage, and after 4 weeks of modified-atmosphere storage.

\begin{tabular}{|c|c|c|c|c|c|c|c|c|c|}
\hline \multirow[b]{2}{*}{ Storage } & \multirow[b]{2}{*}{ Year } & \multicolumn{6}{|c|}{ Cultivar } & \multirow[b]{2}{*}{$\begin{array}{c}\text { Storage } \\
\text { means }\end{array}$} & \multirow[b]{2}{*}{$\begin{array}{l}\text { Year } \\
\text { mean }\end{array}$} \\
\hline & & Bing & Celeste & Cristalina & Samba & $\begin{array}{c}\text { Sandra } \\
\text { Rose }\end{array}$ & Santina & & \\
\hline \multicolumn{10}{|c|}{ Stem shrivel } \\
\hline \multirow[t]{2}{*}{ Harvest } & 1997 & $4.0 \mathrm{a}^{\mathrm{y}}$ & $4.0 \mathrm{a}$ & $4.0 \mathrm{a}$ & $4.0 \mathrm{a}$ & $4.0 \mathrm{a}$ & $4.0 \mathrm{a}$ & \multirow[t]{2}{*}{$3.9 \mathrm{~A}^{\mathrm{x}}$} & $3.3 \mathrm{Z}$ \\
\hline & 1998 & $3.0 \mathrm{efg}$ & $4.0 \mathrm{a}$ & $4.0 \mathrm{a}$ & $3.5 \mathrm{~cd}$ & $3.9 \mathrm{ab}$ & $3.9 \mathrm{ab}$ & & $2.4 \mathrm{Y}$ \\
\hline \multirow[t]{2}{*}{ Air } & 1997 & $2.9 \mathrm{fgh}$ & $2.8 \mathrm{ghi}$ & $2.9 \mathrm{fgh}$ & $3.1 \mathrm{efg}$ & $3.0 \mathrm{efg}$ & $2.4 \mathrm{j}$ & \multirow[t]{2}{*}{$2.2 \mathrm{C}$} & \\
\hline & 1998 & $1.1 \mathrm{n}$ & $1.1 \mathrm{n}$ & $2.5 \mathrm{ij}$ & $1.3 \mathrm{mn}$ & $1.8 \mathrm{kl}$ & $1.8 \mathrm{kl}$ & & \\
\hline \multirow{3}{*}{$\begin{array}{l}\text { Modified } \\
\text { atmosphere }\end{array}$} & & & & & & & & \multirow{4}{*}{$2.6 \mathrm{~B}$} & \\
\hline & 1997 & $3.2 \mathrm{def}$ & $3.3 \mathrm{cde}$ & $3.6 \mathrm{bc}$ & $3.3 \mathrm{cde}$ & $2.4 \mathrm{j}$ & $3.0 \mathrm{efg}$ & & \\
\hline & 1998 & $1.3 \mathrm{mn}$ & $2.0 \mathrm{k}$ & $2.9 \mathrm{fgh}$ & $1.6 \mathrm{~lm}$ & $1.7 \mathrm{kl}$ & $2.6 \mathrm{hij}$ & & \\
\hline \multirow[t]{2}{*}{ Cultivar means } & & $2.5 \mathrm{H}$ & $2.9 \mathrm{FG}$ & $3.3 \mathrm{E}$ & $2.8 \mathrm{G}$ & $2.8 \mathrm{G}$ & $2.9 \mathrm{~F}$ & & \\
\hline & \multicolumn{9}{|c|}{ Stem browning } \\
\hline Harvest & 1997 & $3.4 \mathrm{bc}$ & $3.7 \mathrm{ab}$ & $3.8 \mathrm{a}$ & $3.5 \mathrm{abc}$ & $3.3 \mathrm{c}$ & $3.8 \mathrm{a}$ & \multirow[t]{2}{*}{$3.5 \mathrm{~A}$} & $2.9 \mathrm{Z}$ \\
\hline & 1998 & $2.5 \mathrm{efgh}$ & $3.5 \mathrm{abc}$ & $3.7 \mathrm{ab}$ & $3.5 \mathrm{abc}$ & $3.7 \mathrm{ab}$ & $3.5 \mathrm{abc}$ & & $2.7 \mathrm{Y}$ \\
\hline \multirow[t]{2}{*}{ Air } & 1997 & $2.5 \mathrm{efgh}$ & $2.2 \mathrm{hij}$ & $2.6 \mathrm{defg}$ & $2.8 \mathrm{de}$ & 2.4 fghi & $2.7 \mathrm{def}$ & \multirow[t]{2}{*}{$2.3 \mathrm{C}$} & \\
\hline & 1998 & $2.1 \mathrm{ij}$ & 1.11 & $2.7 \mathrm{def}$ & $1.6 \mathrm{k}$ & $2.5 \mathrm{efgh}$ & 2.4 fghi & & \\
\hline \multirow{3}{*}{$\begin{array}{l}\text { Modified } \\
\text { atmosphere }\end{array}$} & & & & & & & & \multirow{4}{*}{$2.6 \mathrm{~B}$} & \\
\hline & 1997 & $2.5 \mathrm{efgh}$ & $2.7 \mathrm{def}$ & $2.8 \mathrm{de}$ & 2.6 defg & $2.6 \mathrm{defg}$ & $2.7 \mathrm{def}$ & & \\
\hline & 1998 & $2.1 \mathrm{ij}$ & $2.3 \mathrm{ghi}$ & $3.3 \mathrm{c}$ & $1.9 \mathrm{jk}$ & 2.4 fghi & $2.9 \mathrm{~d}$ & & \\
\hline \multirow[t]{2}{*}{ Cultivar means } & & $2.5 \mathrm{I}$ & $2.6 \mathrm{HI}$ & $3.2 \mathrm{E}$ & $2.6 \mathrm{HI}$ & $2.8 \mathrm{G}$ & $3.0 \mathrm{~F}$ & & \\
\hline & \multicolumn{9}{|c|}{ Fruit surface pitting } \\
\hline Harvest & 1997 & $3.0 \mathrm{gh}$ & $3.7 \mathrm{ab}$ & $3.6 \mathrm{abc}$ & $3.1 \mathrm{fgh}$ & $3.4 \mathrm{cde}$ & $3.2 \mathrm{efg}$ & \multirow[t]{2}{*}{$3.3 \mathrm{~A}$} & $2.9 \mathrm{Z}$ \\
\hline & 1998 & $3.5 \mathrm{bcd}$ & $3.0 \mathrm{gh}$ & $3.3 \mathrm{def}$ & 3.4 cde & $3.8 \mathrm{a}$ & $3.0 \mathrm{gh}$ & & $2.5 \mathrm{Y}$ \\
\hline \multirow[t]{2}{*}{ Air } & 1997 & $2.9 \mathrm{hi}$ & 2.0 no & $3.0 \mathrm{gh}$ & $3.0 \mathrm{gh}$ & $2.5 \mathrm{jkl}$ & $2.9 \mathrm{hi}$ & \multirow[t]{2}{*}{$2.5 \mathrm{~B}$} & \\
\hline & 1998 & $2.4 \mathrm{kl}$ & $1.8 \mathrm{o}$ & $2.5 \mathrm{jkl}$ & $2.4 \mathrm{kl}$ & 2.0 no & $2.4 \mathrm{kl}$ & & \\
\hline \multirow{3}{*}{$\begin{array}{l}\text { Modified } \\
\quad \text { atmosphere }\end{array}$} & & & & & & & & \multirow{4}{*}{$2.2 \mathrm{C}$} & \\
\hline & 1997 & $2.6 \mathrm{jk}$ & $1.8 \mathrm{o}$ & $3.2 \mathrm{efg}$ & $2.7 \mathrm{ij}$ & $1.8 \mathrm{o}$ & $2.9 \mathrm{hi}$ & & \\
\hline & 1998 & 1.9 no & $1.3 \mathrm{p}$ & $2.3 \mathrm{~lm}$ & $2.1 \mathrm{mn}$ & $1.3 \mathrm{p}$ & $2.3 \mathrm{~lm}$ & & \\
\hline Cultivar means & & $2.7 \mathrm{~F}$ & $2.3 \mathrm{H}$ & $3.0 \mathrm{E}$ & $2.8 \mathrm{~F}$ & $2.4 \mathrm{G}$ & $2.8 \mathrm{~F}$ & & \\
\hline
\end{tabular}

${ }^{2}$ Stem and fruit defect rating: $4=$ unblemished stems or fruit; $3=$ slight defect; $2=$ moderate defect; 1 = severe defect.

${ }^{y}$ Mean separation for cultivar-storage-year combinations for each defect (lowercase letters) by LSD, $P \leq 0.05$.

${ }^{x}$ Mean separation for cultivars, storage types including harvest, or years for each defect (uppercase letters) by LSD, $P \leq 0.05$.

were continuously flushed with air at a rate of $1.5 \mathrm{~L} \cdot \mathrm{h}^{-1}$. The output of each jar was connected to an automated solenoid switching system. Every $5 \mathrm{~min}$, the sampler was advanced to the output from the next jar and the detector was flushed with gas from the new sample. The level of $\mathrm{CO}_{2}$ was detected with $\Delta \mathrm{P}$ infrared instrument [Analytical Development Co., (equipment type = DPIP-CD-1900-0), Hoddesdon, England] and logged by computer. Samples were analyzed over a $24-\mathrm{h}$ period. Rates of $\mathrm{CO}_{2}$ production are expressed as $\mathrm{mmol} \cdot \mathrm{h}^{-1}$.

Data were analyzed in a factorial design with cultivar, treatment (harvest, air storage or MA) and year as main effects for the following observations: change in weight during storage; stem and fruit defect ratings; and fruit quality measurements TSSC, TA, and fruit firmness). When $\mathrm{F}$ values were significant a least significant difference (LSD) $(P=0.05)$ was calculated. The relationship between fruit respiration at harvest and harvest date was examined by regression analysis, as was the relationship between change in TA vs. change in fruit firmness during storage.

\section{Results}

Most interaction terms (cultivar $\times$ treatment, cultivar $\times$ year, treatment $\times$ year, and cultivar $\times$ treatment $\times$ year) were significant for TSSC, TA, fruit firmness and defect ratings of stem and fruit for both Group 1 (except the treatment $\times$ year interaction for TSSC) and
Group 2 cultivars. Weight change of fruit during storage of Group 1 cultivars was influenced only by storage treatment, with fruit stored in air losing 5\% and fruit stored in MA losing $<1 \%$ of their initial weight (data not shown). The weight loss during storage for the Group 2 cultivars was influenced by year, storage treatment, and cultivar (Table 1). In 1997 and 1999, 'Bing' cherries in air storage lost significantly more weight than did all other cultivars $(\approx 6 \%$ and $10 \%$, in 1997 and 1999, respectively). In air storage all cultivars except 'Bing' lost $\approx 2 \%$ of their initial weights in 1997, whereas in 1998, all cultivars lost between $3 \%$ and $5 \%$ of their initial weight. The greatest variation for weight loss among cultivars occurred in 1999. Cherries in MA treatments generally lost $<1 \%$ of their initial weight in all years, except for 'Bing' in 1998.

Susceptibility of the different cultivars to various defects, such as stem shrivel, stem browning, and fruit surface pitting varied from year to year for Group 1 fruit (Table 2). At harvest no stem shrivel was evident in 1997, whereas some shrivel in 'Samba' and 'Bing' was found in 1998. Stems on fruit held in air storage had more shrivel (lower scores) than those on fruit from MA, except for 'Sandra Rose' in 1997. Stem shrivel was greater in 1998 than in 1997. In both years, stems began to brown at harvest. Stem browning in 1997 was similar for all cultivars except 'Celeste', whether they were in air storage or MA. In 1998, however, stems of
'Santina', 'Celeste', and 'Cristalina', browned more in air storage than in MA. Surface pitting was also evident on fruit at harvest in both years. For some cultivars, such as 'Bing', 'Celeste', 'Samba', and 'Sandra Rose', more pitting occurred after MA than after air storage.

Defect ratings also varied from year to year with the Group 2 fruit (Table 3). At harvest, no stem shrivel was found in 1997 and 1999, but 'Sweetheart' and 'Bing' had more stem shrivel than did other cultivars in 1998. In 1997 stem shrivel was similar on all cultivars except 'Bing' whether fruit were stored in air or MA. In 1998 all cultivars except 'Bing' had more stem shrivel when stored in air than in MA. Stem shrivel in 'Skeena' and 'Sweetheart' was not affected by method of storage in 1999. Stem browning was evident on fruit at harvest. In 1997 method of storage affected browning only in 'Lapins'; browning increased during storage. In 1998 browning of stems was not affected by storage regime except in 'Sonata' and 'Sweetheart'. In 1999 more stem browning occurred in air than in MA in all cultivars except 'Bing'. Surface pitting was also evident for some cultivars at harvest in all 3 years. Only in 1997 did most cultivars have more pitting after MA than after air storage.

Except for 'Santina' in both years, 'Celeste' and 'Cristalina' in 1997 and 'Samba' and 'Bing' in 1998, TSSC did not decrease during storage (Table 4). In all these cases air-stored fruit had higher TSSC levels than 
Table 3. Stem and fruit defect ratings $\mathrm{s}^{\mathrm{z}}$ of sweet cherry cultivars in 1997, 1998, and 1999 at harvest, after 2 weeks of air storage, and after 4 weeks of modified-atmosphere storage.

\begin{tabular}{|c|c|c|c|c|c|c|c|c|}
\hline \multirow[b]{2}{*}{ Storage } & \multirow[b]{2}{*}{ Year } & \multicolumn{5}{|c|}{ Cultivar } & \multirow{2}{*}{$\begin{array}{c}\text { Storage } \\
\text { means }\end{array}$} & \multirow{2}{*}{$\begin{array}{l}\text { Year } \\
\text { means }\end{array}$} \\
\hline & & Bing & Lapins & Skeena & Sonata & Sweetheart & & \\
\hline \multicolumn{9}{|c|}{ Stem shrivel } \\
\hline \multirow[t]{3}{*}{ Harvest } & 1997 & $4.0 \mathrm{a}^{y}$ & $4.0 \mathrm{a}$ & $4.0 \mathrm{a}$ & $4.0 \mathrm{a}$ & $4.0 \mathrm{a}$ & \multirow[t]{3}{*}{$3.9 \mathrm{~A}^{\mathrm{x}}$} & $3.1 \mathrm{~B}$ \\
\hline & 1998 & $3.0 \mathrm{de}$ & $3.8 \mathrm{ab}$ & $3.9 \mathrm{a}$ & $3.9 \mathrm{a}$ & $3.6 \mathrm{bc}$ & & $2.3 \mathrm{C}$ \\
\hline & 1999 & $4.0 \mathrm{a}$ & $4.0 \mathrm{a}$ & $4.0 \mathrm{a}$ & $4.0 \mathrm{a}$ & $4.0 \mathrm{a}$ & & $3.7 \mathrm{~A}$ \\
\hline \multirow[t]{3}{*}{ Air } & 1997 & $2.9 \mathrm{ef}$ & $2.5 \mathrm{hi}$ & $2.4 \mathrm{ij}$ & $2.8 \mathrm{efg}$ & 2.6 ghi & \multirow[t]{3}{*}{$2.4 \mathrm{C}$} & \\
\hline & 1998 & $1.1 \mathrm{n}$ & $1.4 \mathrm{~m}$ & $1.4 \mathrm{~m}$ & $1.4 \mathrm{~m}$ & $1.1 \mathrm{n}$ & & \\
\hline & 1999 & $2.5 \mathrm{hi}$ & $3.5 \mathrm{c}$ & $3.8 \mathrm{ab}$ & $3.5 \mathrm{c}$ & $3.6 \mathrm{bc}$ & & \\
\hline \multirow{3}{*}{$\begin{array}{l}\text { Modified } \\
\quad \text { atmosphere }\end{array}$} & & & & & & & \multirow{4}{*}{$2.8 \mathrm{~B}$} & \\
\hline & $\begin{array}{l}1997 \\
1998\end{array}$ & $\begin{array}{l}3.2 \mathrm{~d} \\
1.3 \mathrm{mn}\end{array}$ & $2.4 \mathrm{ij}$ & $2.5 \mathrm{hi}$ & $\begin{array}{l}3.0 \mathrm{de} \\
2.5 \mathrm{hi}\end{array}$ & $2.7 \mathrm{fgh}$ & & \\
\hline & $\begin{array}{l}1998 \\
1999\end{array}$ & $\begin{array}{l}1.3 \mathrm{mn} \\
3.6 \mathrm{bc}\end{array}$ & $\begin{array}{l}2.1 \mathrm{k} \\
3.8 \mathrm{ab}\end{array}$ & $\begin{array}{l}1.81 \\
3.8 \mathrm{ab}\end{array}$ & $\begin{array}{l}2.5 \mathrm{hi} \\
3.9 \mathrm{a}\end{array}$ & $\begin{array}{l}2.2 \mathrm{jk} \\
3.8 \mathrm{ab}\end{array}$ & & \\
\hline \multicolumn{2}{|l|}{ Cultivar means } & $2.8 \mathrm{C}$ & $3.1 \mathrm{~B}$ & $3.1 \mathrm{~B}$ & $3.2 \mathrm{~A}$ & $3.1 \mathrm{~B}$ & & \\
\hline \multicolumn{9}{|c|}{ Stem browning } \\
\hline \multirow[t]{3}{*}{ Harvest } & 1997 & 3.4 bcde & 3.4 bcde & $3.1 \mathrm{efgh}$ & $3.1 \mathrm{efgh}$ & $3.1 \mathrm{efgh}$ & \multirow[t]{3}{*}{$3.3 \mathrm{~A}$} & $2.5 \mathrm{~B}$ \\
\hline & 1998 & $2.5 \mathrm{jkl}$ & $3.6 \mathrm{abc}$ & $3.6 \mathrm{abc}$ & 3.4 bcde & $3.5 \mathrm{abcd}$ & & $2.4 \mathrm{C}$ \\
\hline & 1999 & $3.8 \mathrm{a}$ & $3.7 \mathrm{ab}$ & $3.2 \mathrm{defg}$ & 3.0 fghi & $3.2 \mathrm{defg}$ & & $2.8 \mathrm{~A}$ \\
\hline \multirow[t]{3}{*}{ Air } & 1997 & $2.5 \mathrm{jkl}$ & $2.4 \mathrm{klm}$ & $2.1 \mathrm{mno}$ & $2.1 \mathrm{mno}$ & 2.0 no & \multirow[t]{3}{*}{$2.0 \mathrm{C}$} & \\
\hline & 1998 & $2.1 \mathrm{mno}$ & 1.9 nop & 1.8 op & $1.3 \mathrm{q}$ & $1.6 \mathrm{pq}$ & & \\
\hline & 1999 & $2.9 \mathrm{ghi}$ & $2.5 \mathrm{jkl}$ & $1.6 \mathrm{pq}$ & $1.4 \mathrm{q}$ & $2.5 \mathrm{jkl}$ & & \\
\hline \multirow{4}{*}{$\begin{array}{l}\text { Modified } \\
\text { atmosphere }\end{array}$} & & & & & & & \multirow{5}{*}{$2.4 \mathrm{~B}$} & \\
\hline & 1997 & $2.5 \mathrm{jkl}$ & 2.0 no & 2.0 no & $2.1 \mathrm{mno}$ & $2.2 \mathrm{lmn}$ & & \\
\hline & 1998 & $2.1 \mathrm{mno}$ & $2.2 \mathrm{lmn}$ & $2.1 \mathrm{mno}$ & 1.9 nop & $2.1 \mathrm{mno}$ & & \\
\hline & 1999 & $2.8 \mathrm{ij}$ & $3.3 \mathrm{def}$ & $2.7 \mathrm{ijk}$ & $2.4 \mathrm{klm}$ & $3.1 \mathrm{efgh}$ & & \\
\hline \multicolumn{2}{|l|}{ Cultivar means } & $2.7 \mathrm{~A}$ & $2.8 \mathrm{~A}$ & $2.5 \mathrm{C}$ & $2.3 \mathrm{D}$ & $2.6 \mathrm{~B}$ & & \\
\hline \multicolumn{9}{|c|}{ Fruit surface pitting } \\
\hline \multirow[t]{3}{*}{ Harvest } & 1997 & 3.0 efg & $3.6 \mathrm{bc}$ & $3.7 \mathrm{ab}$ & $3.5 \mathrm{bc}$ & $3.6 \mathrm{bc}$ & \multirow{3}{*}{$3.4 \mathrm{~A}$} & $2.9 \mathrm{~A}$ \\
\hline & 1998 & $3.5 \mathrm{bc}$ & $3.1 \mathrm{ef}$ & $3.4 \mathrm{~cd}$ & $3.0 \mathrm{efg}$ & $2.7 \mathrm{hij}$ & & $2.5 \mathrm{C}$ \\
\hline & 1999 & $3.9 \mathrm{a}$ & $3.9 \mathrm{a}$ & $3.2 \mathrm{de}$ & $3.0 \mathrm{efg}$ & $3.1 \mathrm{ef}$ & & $2.8 \mathrm{~B}$ \\
\hline \multirow[t]{3}{*}{ Air } & 1997 & $2.9 \mathrm{fgh}$ & $3.1 \mathrm{ef}$ & $2.9 \mathrm{fgh}$ & $2.9 \mathrm{fgh}$ & $2.6 \mathrm{ijk}$ & \multirow[t]{3}{*}{$2.5 \mathrm{~B}$} & \\
\hline & 1998 & $2.4 \mathrm{klm}$ & $2.0 \mathrm{opq}$ & $2.8 \mathrm{ghi}$ & 2.1 nop & $1.7 \mathrm{r}$ & & \\
\hline & 1999 & $2.9 \mathrm{fgh}$ & $2.3 \mathrm{lmn}$ & $2.6 \mathrm{ijk}$ & $2.3 \mathrm{lmn}$ & $2.3 \mathrm{lmn}$ & & \\
\hline Modified & & & & & & & & \\
\hline atmosphere & 1997 & $2.6 \mathrm{ijk}$ & $2.3 \mathrm{lmn}$ & $2.6 \mathrm{ijk}$ & 2.1 nop & $2.6 \mathrm{ijk}$ & $2.4 \mathrm{C}$ & \\
\hline & 1998 & $1.9 \mathrm{pqr}$ & $2.2 \mathrm{mno}$ & $2.7 \mathrm{hij}$ & $1.9 \mathrm{pqr}$ & $1.8 \mathrm{qr}$ & & \\
\hline & 1999 & 2.1 nop & $2.8 \mathrm{ghi}$ & $2.5 \mathrm{jkl}$ & $2.5 \mathrm{jkl}$ & $2.6 \mathrm{ijk}$ & & \\
\hline Cultivar means & & $2.8 \mathrm{~B}$ & $2.8 \mathrm{~B}$ & $2.9 \mathrm{~A}$ & $2.6 \mathrm{C}$ & $2.6 \mathrm{C}$ & & \\
\hline
\end{tabular}

${ }^{2}$ Stem and fruit defect rating: 4 = unblemished stems or fruit; $3=$ slight defect; 2 = moderate defect; 1 = severe defect.

${ }^{y}$ Mean separation for cultivar-storage-year combinations for each defect (lowercase letters) by LSD, $P \leq 0.05$.

${ }^{\times}$Mean separation for cultivars, storage types including harvest, or years for each defect (uppercase letters) by LSD, $P \leq 0.05$.

at harvest. The TA followed a similar pattern in the 2 years; 'Santina', 'Celeste', and 'Cristalina' had lower TA than did 'Samba', 'Sandra Rose', and 'Bing'. Except for 'Celeste' in 1997, fruit from MA had lower TA levels than did freshly harvested or airstored fruit in both years. Generally, changes in fruit firmness differed in 1997 and 1998. In 1997 , firmness declined during storage in all cultivars except 'Sandra Rose'; in 1998, firmness of 'Santina', 'Cristalina', and 'Sandra Rose' did not change during air storage.

For most Group 2 cultivars TSSC did not change during storage in 1997 (Table 5). The TSSC increased during air storage of all cultivars except 'Lapins' in 1998, but only in 'Skeena' and 'Bing' in 1999. The TA levels decreased with time in storage for all cultivars. In 1997 and 1998 'Bing' appeared to have the smallest decrease in TA. Air-stored fruit tended to have lower firmness regardless of cultivar, whereas firmness of fruit in MA either remained constant or increased.

The respiration rate of fruit at harvest decreased with chronological picking date in both years (Fig. 1), but respiration rates were not correlated with fruit quality characteristics after storage (data not shown). Initial TA and fruit quality after storage were not related

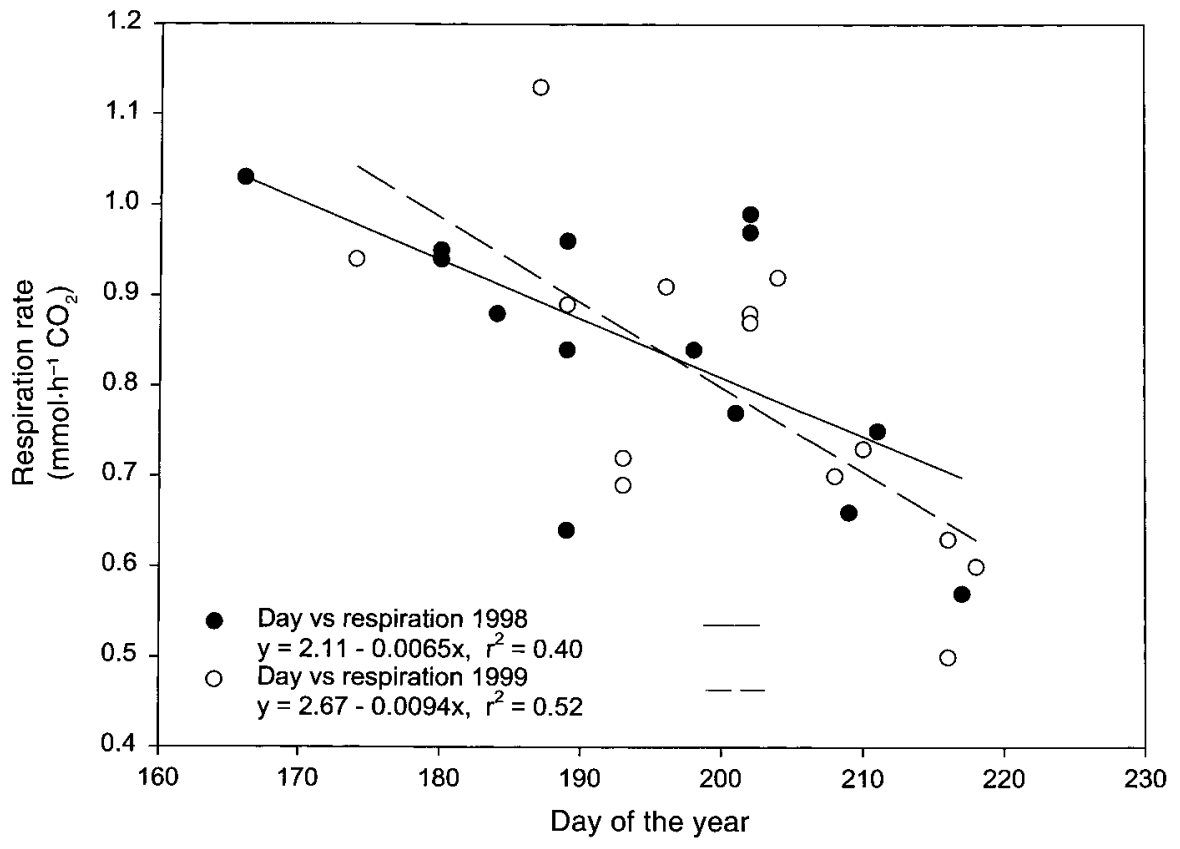

Fig. 1. Respiration of fruits of 15 sweet cherry cultivars harvested and measured at commercial maturity. 
Table 4. Total soluble solids, titratable acidity, and fruit firmness of sweet cherry cultivars in 1997 and 1998 at harvest, after 2 weeks air storage and after 4 weeks modified-atmosphere storage.

\begin{tabular}{|c|c|c|c|c|c|c|c|c|c|}
\hline \multirow[b]{2}{*}{ Storage } & \multirow[b]{2}{*}{ Year } & \multicolumn{6}{|c|}{ Cultivar } & \multirow[b]{2}{*}{$\begin{array}{c}\text { Storage } \\
\text { means }\end{array}$} & \multirow[b]{2}{*}{$\begin{array}{c}\text { Year } \\
\text { means }\end{array}$} \\
\hline & & Bing & Celeste & Cristalina & Samba & $\begin{array}{l}\text { Sandra } \\
\text { Rose }\end{array}$ & Santina & & \\
\hline \multicolumn{10}{|c|}{ Soluble solids (\%) } \\
\hline \multirow[t]{2}{*}{ Harvest } & 1997 & $17.5 \mathrm{no}^{\mathrm{z}}$ & 19.2 ghij & $18.3 \mathrm{klm}$ & 19.5 efgh & $19.3 \mathrm{fghi}$ & $15.7 \mathrm{r}$ & $18.0 \mathrm{~B}^{\mathrm{y}}$ & $18.6 \mathrm{Z}$ \\
\hline & 1998 & $19.6 \mathrm{efg}$ & $17.7 \mathrm{mno}$ & $16.1 \mathrm{qr}$ & $18.6 \mathrm{ijk}$ & $20.4 \mathrm{abcd}$ & $14.7 \mathrm{~s}$ & & $18.2 \mathrm{Y}$ \\
\hline \multirow[t]{2}{*}{ Air } & 1997 & $18.0 \mathrm{lmn}$ & $21.0 \mathrm{a}$ & 19.3 fghi & $19.6 \mathrm{efg}$ & 20.1 cde & $17.1 \mathrm{op}$ & $18.9 \mathrm{~A}$ & \\
\hline & 1998 & $20.9 \mathrm{ab}$ & $18.4 \mathrm{kl}$ & $16.6 \mathrm{pq}$ & $19.6 \mathrm{efg}$ & $20.8 \mathrm{abc}$ & $15.9 \mathrm{qr}$ & & \\
\hline \multicolumn{10}{|l|}{ Modified } \\
\hline \multirow[t]{2}{*}{ atmosphere } & 1997 & $18.1 \mathrm{lmn}$ & 19.0 hijk & 17.5 no & $19.6 \mathrm{efg}$ & 20.2 bcde & $15.8 \mathrm{r}$ & $18.1 \mathrm{~B}$ & \\
\hline & 1998 & $20.0 \mathrm{def}$ & $18.1 \mathrm{lmn}$ & $16.0 \mathrm{gr}$ & $18.5 \mathrm{jkl}$ & $20.5 \mathrm{abcd}$ & $14.4 \mathrm{~s}$ & & \\
\hline \multirow[t]{2}{*}{ Cultivar means } & & $19.0 \mathrm{FG}$ & $18.9 \mathrm{G}$ & $17.3 \mathrm{H}$ & $19.2 \mathrm{~F}$ & $20.2 \mathrm{E}$ & $15.6 \mathrm{I}$ & & \\
\hline & \multicolumn{9}{|c|}{ Titratable acidity $^{x}$} \\
\hline \multirow[t]{2}{*}{ Harvest } & 1997 & $8.8 \mathrm{j}$ & $6.9 \mathrm{mn}$ & $6.4 \mathrm{n}$ & $10.4 \mathrm{f}$ & $10.6 \mathrm{f}$ & $7.7 \mathrm{kl}$ & $10.0 \mathrm{~A}$ & $8.2 \mathrm{Y}$ \\
\hline & 1998 & $14.1 \mathrm{a}$ & $8.9 \mathrm{ij}$ & $8.6 \mathrm{j}$ & $13.5 \mathrm{bc}$ & $13.5 \mathrm{bc}$ & $9.4 \mathrm{hi}$ & & $10.9 \mathrm{Z}$ \\
\hline \multirow[t]{2}{*}{ Air } & 1997 & $8.6 \mathrm{j}$ & $7.4 \mathrm{~lm}$ & $6.9 \mathrm{mn}$ & $10.4 \mathrm{f}$ & $10.6 \mathrm{f}$ & $7.8 \mathrm{kl}$ & $9.8 \mathrm{~A}$ & \\
\hline & 1998 & $14.0 \mathrm{ab}$ & $10.0 \mathrm{~g}$ & $6.9 \mathrm{mn}$ & $13.6 \mathrm{abc}$ & $13.2 \mathrm{c}$ & $8.8 \mathrm{j}$ & & \\
\hline \multirow{3}{*}{$\begin{array}{l}\text { Modified } \\
\text { atmosphere }\end{array}$} & & & & & & & & & \\
\hline & 1997 & $8.0 \mathrm{k}$ & $7.0 \mathrm{~m}$ & $5.2 \mathrm{o}$ & $9.6 \mathrm{gh}$ & 9.1 hij & $6.4 \mathrm{n}$ & $8.8 \mathrm{~B}$ & \\
\hline & 1998 & $13.5 \mathrm{bc}$ & $9.5 \mathrm{gh}$ & $6.4 \mathrm{n}$ & $12.5 \mathrm{~d}$ & $11.6 \mathrm{e}$ & 7.7 kl & & \\
\hline \multirow[t]{2}{*}{ Cultivar means } & & $11.2 \mathrm{~F}$ & $8.3 \mathrm{G}$ & $6.7 \mathrm{I}$ & $11.7 \mathrm{E}$ & $11.5 \mathrm{E}$ & $8.0 \mathrm{H}$ & & \\
\hline & \multicolumn{9}{|c|}{ Fruit firmness $^{w}\left(g \cdot \mathrm{mm}^{-1}\right)$} \\
\hline \multirow[t]{2}{*}{ Harvest } & 1997 & $188 \mathrm{~h}$ & $203 \mathrm{fg}$ & $249 \mathrm{a}$ & $233 \mathrm{bcd}$ & $134 \mathrm{~m}$ & 227 cde & $191 \mathrm{~B}$ & $193 \mathrm{Z}$ \\
\hline & 1998 & $154 \mathrm{jk}$ & $193 \mathrm{fgh}$ & $193 \mathrm{fgh}$ & $243 \mathrm{ab}$ & $114 n$ & $165 \mathrm{ijk}$ & & $180 \mathrm{Y}$ \\
\hline \multirow[t]{2}{*}{ Air } & 1997 & $153 \mathrm{kl}$ & $140 \mathrm{~m}$ & $192 \mathrm{gh}$ & $201 \mathrm{fg}$ & $141 \mathrm{~lm}$ & $166 \mathrm{ij}$ & $164 \mathrm{C}$ & \\
\hline & 1998 & $135 \mathrm{~m}$ & $167 \mathrm{i}$ & $191 \mathrm{gh}$ & $202 \mathrm{fg}$ & $121 \mathrm{n}$ & $159 \mathrm{ijk}$ & & \\
\hline \multirow{3}{*}{$\begin{array}{l}\text { Modified } \\
\text { atmosphere }\end{array}$} & & & & & & & & & \\
\hline & 1997 & $181 \mathrm{~h}$ & $221 \mathrm{de}$ & $221 \mathrm{de}$ & $254 \mathrm{a}$ & $162 \mathrm{ijk}$ & $202 \mathrm{fg}$ & $204 \mathrm{~A}$ & \\
\hline & 1998 & $159 \mathrm{ijk}$ & $205 \mathrm{f}$ & $234 \mathrm{bc}$ & $251 \mathrm{a}$ & $139 \mathrm{~m}$ & $219 \mathrm{e}$ & & \\
\hline Cultivar means & & $162 \mathrm{H}$ & $188 \mathrm{C}$ & $213 \mathrm{~F}$ & $231 \mathrm{E}$ & $135 \mathrm{I}$ & $190 \mathrm{G}$ & & \\
\hline
\end{tabular}

${ }^{2}$ Mean separation for cultivar-storage-year combinations for each measurement (lowercase letters) by LSD, $P \leq 0.05$.

${ }^{y}$ Mean separation for cultivars, storage type including harvest, or years for each measurement (uppercase letters) by LSD, $P \leq 0.05$.

${ }^{x} \mathrm{~mL} 0.1 \mathrm{~N} \mathrm{NaOH}$ required to adjust $\mathrm{pH}$ of $10 \mathrm{~mL}$ juice to 8.1 .

${ }^{w}$ Weight required to compress cherry $1 \mathrm{~mm}$.

(data not shown), although fruit firmness increased as TA decreased (data not shown).

\section{Discussion}

Weight loss differed among cultivars held in air but not in MA, but the differences were not consistent from year to year. 'Bing' tended to have the greatest weight loss in all years. Sekse (1988) reported that weight loss was influenced by the RH in the storage atmosphere, and our results appear to confirm this, with greater weight loss in air storage than in MA. Beaudry and Lakakul (1995) reported that the greatest benefit of packaging is a greater RH around the product. Sharkey and Peggie (1984) reported that sweet cherries stored at high RH were firmer, and had brighter skin and less stem shrivel, than cherries stored at lower $\mathrm{RH}$ at the same temperature.

Stem and fruit defects were affected by cultivar, year, and storage condition. Some cultivars had defects at harvest, a feature noted by Facteau and Rowe (1979), who reported that surface pitting was evident on some freshly harvested cherries. Greater incidences of defects in 1998 than in other years was probably due to the extremely warm season in 1998. July monthly means were $19.2,23.8$, and $19.6^{\circ} \mathrm{C}$, for 1997,1998 , and 1999, respectively. Among the Group 1 cultivars, 'Cristalina' appeared to have good quality following storage (firm, bright, shiny fruit and fresh appearing stems with absence of defects), comparable to or exceeding that of 'Bing', which is the industry standard. 'Santina' was another cultivar that appeared to maintain good fruit quality during storage. 'Celeste' and 'Sandra Rose' had quality ratings lower than 'Bing'. No cultivar in Group 2 was superior to 'Bing'.

The concentration of TSSC generally increased during air storage of both Groups 1 and 2 , with an average increase of $<1 \%$. The dehydration of the fruit during the 2 weeks of air storage could explain the increase in the sugar concentration.

The greatest change in fruit quality measurements during storage was in firmness. Fruit in air storage became softer, whereas fruit in MA tended to be similar in firmness or firmer than at harvest. Increased flesh firmness has been reported in both MA and CA storage (Chen et al., 1981; Meheriuk et al., 1997). Sharkey and Peggie (1984) reported greater firmness of two cherry cultivars when stored at $95 \%$ to $99 \%$ relative humidity than at $90 \%$ to $94 \%$.

Respiration rates of sweet cherry cultivars at harvest may be an indicator of their storage potential. Respiration rate of five red raspberry cultivars (Rubus idaeus L.) was correlated positively with rot, weight loss and change in TSSC, and negatively with changes in firmness (Robbins et al., 1989). Our work does not support such a correlation for sweet cherries. Respiration rate of fruit at harvest was not related to any of the quality measurements taken after storage. The only consistent relationship was that later matur- ing cultivars had lower respiration rates than early maturing ones. There also was a negative relationship between fruit firmness and TA during storage; Meheriuk et al. (1997) found a similar relationship for 'Sweetheart' cherry.

Cultivars varied from year to year in their response to the storage regimes. Generally, fruit stored in MAP bags appeared to be of better quality than those stored in air. Fruit quality after storage of some cultivars, such as 'Cristalina' and 'Samba' from Group 1 and 'Skeena' and 'Sonata' from Group 2, was comparable to or better than that of 'Bing', the main industry cultivar.

\section{Literature Cited}

Beaudry, R. and R. Lakakul. 1995. Basic principles of modified atmosphere packaging, p. 713. In: E. Kupferman and H. Waelti (eds.). Tree fruit postharvest journal. Coop. Ext. Wash. State Univ., Wenatchee.

Brown, S.K. and M.C. Bourne. 1988. Assessments of components of fruit firmness in selected sweet cherry genotypes. HortScience 23:902904.

Chen, P.M., W.M. Mellenthin, S.B. Kelly, and T.J. Facteau. 1981. Effects of low oxygen and temperature on quality retention of 'Bing' cherries during storage. J. Amer. Soc. Hort. Sci. 106:533-535.

Drake, S.R. and J.K. Fellman. 1987. Indicators of maturity and storage quality of 'Rainier' sweet cherry. HortScience 22:283-285.

Facteau, T.J. and K.E. Rowe. 1979. Factors associ- 
Table 5. Total soluble solids, titratable acidity, and fruit firmness of late sweet cherry cultivars in 1997, 1998, and 1999 at harvest, after 2 weeks Table 5. Total soluble solids, tritatable acidity, and fruit firmness of late sweet cherry cultivars in 1997, 1998, and 1999 at harvest, after 2 weeks air storage, and after 4 weeks modified-atmosphere storage.

\begin{tabular}{|c|c|c|c|c|c|c|c|c|}
\hline \multirow[b]{2}{*}{ Storage } & \multirow[b]{2}{*}{ Year } & \multicolumn{5}{|c|}{ Cultivar } & \multirow{2}{*}{$\begin{array}{r}\text { Storage } \\
\text { means }\end{array}$} & \multirow{2}{*}{$\begin{array}{l}\text { Year } \\
\text { means }\end{array}$} \\
\hline & & Bing & Lapins & Skeena & Sonata & Sweetheart & & \\
\hline \multicolumn{9}{|c|}{ Soluble solids (\%) } \\
\hline \multirow{3}{*}{ Harvest } & 1997 & $17.5 \mathrm{~s}^{\mathrm{z}}$ & $15.7 \mathrm{t}$ & $20.9 \mathrm{defg}$ & $18.1 \mathrm{n}-\mathrm{s}$ & $18.41-\mathrm{r}$ & $19.1 \mathrm{~B}^{\mathrm{y}}$ & $18.4 X$ \\
\hline & 1998 & $19.6 \mathrm{hij}$ & $17.7 \mathrm{rs}$ & $19.1 \mathrm{jkl}$ & 17.9 pqrs & $20.4 \mathrm{fg}$ & & $19.5 \mathrm{Y}$ \\
\hline & 1999 & $21.0 \mathrm{cdef}$ & $19.0 \mathrm{jklm}$ & $20.4 \mathrm{fg}$ & $20.0 \mathrm{hi}$ & $21.7 \mathrm{bc}$ & & $20.5 \mathrm{Z}$ \\
\hline \multirow[t]{3}{*}{ Air } & 1997 & $18.0 \mathrm{o}-\mathrm{s}$ & $15.9 \mathrm{t}$ & $21.3 \mathrm{~cd}$ & $19.3 \mathrm{ijk}$ & $19.0 \mathrm{jklm}$ & $20.0 \mathrm{~A}$ & \\
\hline & 1998 & 20.9 defg & $18.5 \mathrm{l}-\mathrm{q}$ & $20.2 \mathrm{gh}$ & $18.8 \mathrm{klmn}$ & $22.3 \mathrm{ab}$ & & \\
\hline & 1999 & $23.4 \mathrm{a}$ & $19.0 \mathrm{jklm}$ & $22.1 \mathrm{ab}$ & $20.5 \mathrm{efg}$ & 21.2 cde & & \\
\hline \multirow{4}{*}{$\begin{array}{l}\text { Modified } \\
\text { atmosphere }\end{array}$} & & & & & & & & \\
\hline & 1997 & $18.1 \mathrm{n}-\mathrm{s}$ & $15.9 \mathrm{t}$ & $20.9 \mathrm{defg}$ & $18.3 \mathrm{~m}-\mathrm{r}$ & $18.7 \mathrm{k}-\mathrm{o}$ & $19.1 \mathrm{~B}$ & \\
\hline & 1998 & $20.0 \mathrm{hi}$ & $17.7 \mathrm{rs}$ & $18.6 \mathrm{k}-\mathrm{p}$ & $18.7 \mathrm{k}-\mathrm{o}$ & $21.5 \mathrm{~cd}$ & & \\
\hline & 1999 & $20.4 \mathrm{fg}$ & $17.8 \mathrm{qrs}$ & $20.3 \mathrm{fgh}$ & $19.3 \mathrm{ijk}$ & 20.8 defg & & \\
\hline \multicolumn{2}{|l|}{ Cultivar means } & $19.9 \mathrm{~F}$ & $17.5 \mathrm{H}$ & $20.4 \mathrm{E}$ & $19.0 \mathrm{G}$ & $20.4 \mathrm{E}$ & & \\
\hline \multicolumn{9}{|c|}{ Titratable acidity $^{x}$} \\
\hline \multirow[t]{3}{*}{ Harvest } & 1997 & $8.8 \mathrm{tu}$ & $9.5 \mathrm{~s}$ & $10.4 \mathrm{p}$ & $13.4 \mathrm{k}$ & 11.6 no & $13.3 \mathrm{~A}$ & $10.1 \mathrm{C}$ \\
\hline & 1998 & $14.1 \mathrm{fghi}$ & $11.3 \mathrm{o}$ & 12.71 & $17.2 \mathrm{a}$ & $14.7 \mathrm{de}$ & & $13.1 \mathrm{~B}$ \\
\hline & 1999 & $16.4 \mathrm{bc}$ & $11.2 \mathrm{o}$ & $14.6 \mathrm{def}$ & $17.2 \mathrm{a}$ & $15.9 \mathrm{c}$ & & $14.1 \mathrm{~A}$ \\
\hline \multirow[t]{3}{*}{ Air } & 1997 & $8.6 \mathrm{u}$ & $8.6 \mathrm{u}$ & $10.1 \mathrm{pqr}$ & $13.6 \mathrm{ijk}$ & $10.3 \mathrm{pq}$ & $12.6 \mathrm{~B}$ & \\
\hline & 1998 & 14.0 ghij & $10.6 \mathrm{p}$ & $12.1 \mathrm{mn}$ & $16.1 \mathrm{c}$ & $13.5 \mathrm{jk}$ & & \\
\hline & 1999 & $16.0 \mathrm{c}$ & $9.8 \mathrm{qrs}$ & $14.9 \mathrm{~d}$ & $16.8 \mathrm{ab}$ & 14.1 fghi & & \\
\hline \multirow{4}{*}{$\begin{array}{l}\text { Modified } \\
\text { atmosphere }\end{array}$} & & & & & & & & \\
\hline & 1997 & $8.0 \mathrm{v}$ & $7.8 \mathrm{v}$ & $9.3 \mathrm{st}$ & $12.6 \mathrm{~lm}$ & $9.4 \mathrm{~s}$ & $11.5 \mathrm{C}$ & \\
\hline & 1998 & $13.5 \mathrm{jk}$ & $10.2 \mathrm{pq}$ & $10.3 \mathrm{pq}$ & $14.3 \mathrm{efgh}$ & $12.3 \mathrm{~lm}$ & & \\
\hline & 1999 & 13.9 hijk & $9.6 \mathrm{rs}$ & $12.4 \mathrm{~lm}$ & $14.5 \mathrm{defg}$ & $13.7 \mathrm{ijk}$ & & \\
\hline \multicolumn{2}{|l|}{ Cultivar means } & $12.6 \mathrm{G}$ & $9.8 \mathrm{I}$ & $11.9 \mathrm{H}$ & $15.1 \mathrm{E}$ & $12.8 \mathrm{~F}$ & & \\
\hline \multirow{4}{*}{ Harvest } & & & Fruit & $n e s s^{w}(g \cdot m m$ & & & & \\
\hline & 1997 & 188 qrst & $183 \mathrm{r}-\mathrm{V}$ & $233 \mathrm{hi}$ & 203 mnop & $288 \mathrm{~d}$ & $217 \mathrm{~B}$ & $212 \mathrm{~B}$ \\
\hline & 1998 & $154 \mathrm{x}$ & 188 qrst & $224 \mathrm{ijk}$ & 198 nopq & 194 opqr & & $190 \mathrm{C}$ \\
\hline & 1999 & 176 tuv & $215 \mathrm{klm}$ & $257 \mathrm{fg}$ & $277 \mathrm{~d}$ & $275 \mathrm{de}$ & & 244 A \\
\hline \multirow[t]{3}{*}{ Air } & 1997 & $153 x$ & $183 \mathrm{r}-\mathrm{v}$ & 203 mnop & $187 \mathrm{q}-\mathrm{u}$ & $245 \mathrm{gh}$ & $189 \mathrm{C}$ & \\
\hline & 1998 & $135 \mathrm{y}$ & $171 \mathrm{vw}$ & 190 pqrs & 178 stuv & $186 \mathrm{q}-\mathrm{u}$ & & \\
\hline & 1999 & $139 \mathrm{y}$ & 174 uv & $214 \mathrm{klm}$ & $210 \mathrm{lmn}$ & 263 ef & & \\
\hline \multirow{4}{*}{$\begin{array}{l}\text { Modified } \\
\text { atmosphere }\end{array}$} & & & & & & & & \\
\hline & 1997 & $181 \mathrm{r}-\mathrm{v}$ & 203 mnop & $229 \mathrm{ij}$ & $219 \mathrm{jkl}$ & $285 \mathrm{~d}$ & $240 \mathrm{~A}$ & \\
\hline & 1998 & $159 \mathrm{wx}$ & 189 qrst & $243 \mathrm{~h}$ & $219 \mathrm{jkl}$ & $218 \mathrm{jkl}$ & & \\
\hline & 1999 & 204 mno & $257 \mathrm{fg}$ & $335 \mathrm{a}$ & $302 \mathrm{c}$ & $361 \mathrm{a}$ & & \\
\hline Cultivar means & & $165 \mathrm{I}$ & $196 \mathrm{H}$ & $237 \mathrm{~F}$ & $221 \mathrm{G}$ & $257 \mathrm{E}$ & & \\
\hline
\end{tabular}

${ }^{2}$ Mean separation for cultivar-storage-year combinations for each measurement (lowercase letters) by LSD, $P \leq 0.05$.

${ }^{y}$ Mean separation for cultivars, storage type including harvest, or years for each measurement (uppercase letters) by LSD, $P \leq 0.05$.

${ }^{x} \mathrm{~mL} 0.1 \mathrm{~N} \mathrm{NaOH}$ required to adjust $\mathrm{pH}$ of $10 \mathrm{~mL}$ juice to 8.1 .

wWeight required to compress cherry $1 \mathrm{~mm}$.

ated with surface pitting of sweet cherry. J. Amer. Soc. Hort. Sci. 104:706-710.

Kappel, F., W.D. Lane, R. MacDonald, K. Lapins, and H. Schmid. 1998. 'Santina', 'Sumpaca Celeste', and 'Sumnue Cristalina' sweet cherries. HortScience 33:1087-1089.

Kappel, F., W.D. Lane, R. MacDonald, K. Lapins, and H. Schmid. 2000a. 'Sumste Samba', 'Sandra Rose', and 'Sumleta Sonata' sweet cherries. HortScience 35:152-154.

Kappel, F., W.D. Lane, R. MacDonald, and H. Schmid. 2000b. 'Skeena' sweet cherry. HortScience 35:306-307.

Looney, N.E., A.D. Webster, and E.M. Kupferman. 1996. Harvest and handling sweet cherries for the fresh market, p. 411-441. In: N.E. Looney and A.D. Webster (eds.). Cherries: Crop physiology, production and uses. CAB Intl., Wallingford, UK.

Meheriuk, M., B. Girard, L. Moyls, H.J.T. Beveridge, D.-L. McKenzie, J. Harrison, S. Weintraub, and R. Hocking. 1995. Modified atmosphere packaging of 'Lapins' cherry. Food Res. Intl. 28:238-244.

Meheriuk, M., D.-L. McKenzie, B. Girard, A.L. Moyls, S. Weintaub, R. Hocking, and T. Kopp. 1997. Storage of 'Sweetheart' cherries in sealed plastic film. J. Food Quality 20:189198.

Robbins, J., T. M. Sjulin, and M. Patterson. 1989.
Postharvest storage characteristics and respiration rates in five cultivars of red raspberry. HortScience 24:980-982.

Seske, L. 1988. Storage and storage potential of sweet cherries (Prunus avium L.) as related to respiration rate. Acta Agr. Scand. 38:59-66.

Sharkey, P.J. and I.D. Peggie. 1984. Effects of high-humidity storage on quality, decay and storage life of cherry, lemon and peach fruits. Scientia Hort. 23:181-190.

Singh, B., N.A. Littlefield, and D.K. Salunkhe. 1970. Effects of controlled atmosphere (CA) storage on amino acids, organic acids, sugars, and rate of respiration of 'Lambert' sweet cherry fruit. J. Amer. Soc. Hort. Sci. 95:458-461. 